\title{
Quality of Information and Energy Provisioning (Invited Paper)
}

\author{
Erol Gelenbe FIEEE FACM \\ Member National Academy of Engineering of France \\ Member Academy of Science of Turkey \\ Dept. of Electrical \& Electronic Engineering \\ Imperial College, London SW7 2BT, UK \\ e.gelenbe@imperial.ac.uk
}

\begin{abstract}
The Quality of Information (QoI) can be evaluated through the effect that the information will have on a system which is of critical interest. Although QoI is often be discussed in the context of sensor networks, this paper addresses QoI in a new and important framework: the management of energy distribution. We consider a system that combines constant power generation by some conventional source, together with renewable energy being generated and stored. The consumer has some fixed contract with the conventional energy source and obtains any excess needed energy from storage. We show that imperfections in the interpretation or delivery of information about the consumer's instantaneous needs can lead to measurable deficiencies in energy provisioning. The results are derived using Energy Packet Networks which are a novel approach to modeling energy systems based on queueing theory.
\end{abstract}

\section{INTRODUCTION}

Although we would like to think that the quality of the information (QoI) we receive in our Information-Dense world is high, one can actually argue that it is generally quite poor. For instance, the bias placed by the political tendencies of newspapers on the manner in which major news items are presented in the press has been examined using database theory [4]. Today most of the information that we receive via the web represents the interests of the information providers, rather than our own interests. Advertising via web auctions [16] is fueling the choice of the information the web provides to us, rather than our own needs and expectations. Advertising and sensationalism influence the press and the media, and we are surrounded by paid advertisements wherever we go.

Research publications are often influenced by the simpler problems that we can solve, rather than by the questions that we do not understand and need to address and by difficult open problems. Higher education is increasingly based on a substantial simplification, whether it is in the humanities or in science and technology, so that students can be satisfied into thinking that they understand the material. The high cost of higher education means that language instruction for foreign students is reduced to a purely operational level. The instruction of students in foreign languages that they do not fully understand means that instructors have to simplify the content and impoverish their vocabulary, at the price of a reduced quality of information. Even when language is not a problem, because students have not mastered the language of physics and mathematics, much of engineering education is based on the use of operational identities, such as Ohm's Law, which ignore the actual physical processes that are involved.

In this paper we will address QoI in a novel context related to energy management. With the advent of the Smart Grid, the seamless interaction of energy production and distribution with its control via computers and data networks is crucial. By smart management of conventional (fossil and nuclear) and renewable energy resources, together with energy storage devices, as a function of instantaneous demand, it can reduce the carbon imprint of energy and reduce the overcapacity of the energy transport network. However such a system will be increasingly sensitive to the QoI that it receives, as well as to security risks that propagate through software and the Internet.

\section{Energy Packet Networks}

Energy markets are becoming evolving flexible with consumers becoming able to select choose suppliers, while the latter can dynamically select producers. Carbon takes also provide incentives for renewable energy sources (RES) such as wind and photovoltaic, and smart meters can provide the facilities for fine grained management of energy supply and demand where individual consumers can also become suppliers of energy. The enabling technology in this area are obviously data networks and distributed computer decision systems [3], [7], and recent work has considered how such networks can be optimised to save energy [23], [24]

The use of RES such as solar panels, wind power, tidal flows, hydroelectric sources and geothermal power, come with time variations which are unpredictable and can be aperiodic. While more predictable characteristics are present at the demand, one should also to a certain extent tune or schedule the demand [19] to meet the supply, and forecasting demand [6] using public data [20] has received considerable attention. To smooth out this interaction between dynamic supply and demand, storage systems such as flywheels, batteries of electric vehicles, the uninterruptible power supplies (UPS) of data centres, compressed gas depots (GD), dams and water towers (WT), can be used. Time varying ERS such as wind [21] therefore encourage us to consider storage as a means to 
smooth the supply so as to fit demand [13], [26], [27], [28], [30].

Energy Packets and the analogy between the flow of energy in the electric grid, and of the flow of data in the Internet, has been mentioned as early as 1998 [8] and a hardware implementation of an "power packet" on an indoor power line is described in [34]. Recent ideas have proposed an analogy between the smart grid and the Internet [22] while the implementation of an indoor power distribution network which is inspired from a data network is described in [31].

The concept of an Energy Packet Network (EPN) is inspired by work on neural networks and queuing networks [9], [10] that include both the flow of a commodity, in the present case energy in discrete amounts or energy packets (EP), and its storage and consumption, and it includes a packet network that controls the energy flow, storage and allocation [32], [33]. An EP is a pulse of power and is the basic energy unit of content of such a packet by $E$, small enough to being at the smallest energy needs of the consumer. When a consumer turns of a switch to initiate an energy transfer to a device, we can think of this as a download of a certain number of energy packets from a an "energy server"; in a complex energy network, this download may be received from an energy "buffer" (a storage device) or from a generator. The actual choice of the location from which energy is being received by the consumer may be conducted in some distributed fashion that tries to optimize the instantaneous price, the energy network load, the carbon cost and environmental effect, the energy network losses, and so on. Thus the EPN will actually action two networks simultaneously: the energy network, and the data network that includes the communications and distributed decisions that create the desired effect. Thus an EPN is a fine-grained energy generation, storage and distribution system based on discrete EPs. At the bio-nano level we know that cells can receive energy in the form of sugar molecules, while in digital electronics an EP may be as small as an electron or hole.

An EPN is controlled by one or more Smart Energy Dispatching Centres (SEDCs) which use the consumers' requests, the storage centres' and generators' state, to optimise the energy flows by making use of the energy sources, the existing pricing policies, and satisfying the demand while minimising peak flows. The SEDCs will be based on computer control to make and dispatch decisions via a packet communication network. The aggregate choices made regarding the energy flows can be represented by via routing probabilities for EPs that are modified to optimize system performance, and the EPN also includes the flow, delay and losses of data packets that are used to convey for information and control. While data packets constitute the "triggers" of the mathematical G-network representation [10] of the EPN, the EPs are the "ordinary customers" of the queuing network.

\section{Mathematical RePResentation of AN EPN}

The EPN has a set of energy energy units whose generation rate is $g(i, t)$ in EP/sec at time t, where $g(i, t) \leq G M(i)$ which is its maximum generation rate. The energy sources are either renewable, in which case $i \in R$, or they are conventional $i \in C$. The system has a finite storage capacity $S(j)$ for the j-th ST. Each storage centre has an energy conversion efficiency $0<L(j)<1$ at its input so that on average the arrival of $B^{\prime}(j)$ energy packets to this ST results in the storage of $B(j)=L(j) B^{\prime}(j)$ EPs and it will return energy from storage with an efficiency $j(j)$. Furthermore, in addition to its maximum energy storage capacity, it will also have a maximum rate at which it can store energy, and an energy loss or leakage rate so that if storage is not replenished, the B (j) EPs that it contains will be depleted after some time. In addition, a ST will deliver energy at some rate $\alpha(j, t)<D(j)$, less than the maximum rate $\mathrm{D}(\mathrm{j})$ at which it can deliver energy. The c-th energy consumer module $(\mathrm{CM}) C(c)$ has a consumption rate of $m(c, t)$ in EPs/sec at time t. Some CMs may also store energy locally.

The CMs are connected to an energy distribution network $(\mathrm{EDN})$ represented by a graph, so that link $(\mathrm{u}, \mathrm{v})$ of the directed graph represents a power line that has an energy transport capacity $\mathrm{C}(\mathrm{u}, \mathrm{v})$ which is the maximum amount of energy that can be transferred instantaneously from node $\mathrm{u}$ to node $\mathrm{v}$, and an efficiency $0<c(u, v)<1$ which is the fraction of energy introduced into the link that actually reaches the destination. The nodes of the EDN may be production nodes, CMs, or STs. Each CM will send its energy requests to a Smart Dispatching Centre (SDC) which keeps track of the energy needs and requests in an area and assigns flows from the STs and EGs to the CMs. The SDCs also requests the EGs (including EHs) to replenish the STs. Since the system as a whole depends on constant sensing, monitoring, communication and decision, the computer servers and network equipment will also constantly consume energy and this will be included in the model.

Therefore a queuing network analysis of the system can be constructed having energy production, storage and consumption. The energy flow probabilities are $q(u, v)$, the fraction of energy leaving node $\mathrm{u}$ which is directed towards node $\mathrm{v}$, while $p(u, v)=q(u, v) c(u, v)$ is the probability than an EP leaving $\mathrm{u}$ actually arrives at node v. $Q(v, u)$ is the probability that when node $\mathrm{v}$ consumes or dispatches energy to some other node, then it requests energy from some node $u$. The renewable energy sources (RES) are represented by Poisson flows and the energy consumption of CMs is represented by exponentially distributed random variables of parameter $c(k)$ for the $\mathrm{k}$-th $\mathrm{CM}$. As a result we will write equations that represent the equilibrium behavior of the EPN using G-network theory, and predict both the effective computation and communication rates that result from the needs of the workload and from energy availability at the CMs, and also the amount of energy of different types (including RES) that is composed by the system, the average amount stored, the average amount lost in leakage and transport, and so on.

\section{INFORMATION QUALITY AND ENERGY PROVISIONING}

The performance model we propose focuses on the energy flows in the system and allows us to evaluate many of the quantities of interest including the amount of energy being 
stored in each part of the EPN, the average flow of power on each of the links of the transport network, the probability that a request for power is not satisfied, and the average delay between the time that a request is made until the instant at which the power delivery begins. The model can also be used to estimate the performance risks and enhancements offered by an EPN in terms of performance measures of interest. Since it incorporates both the power transport network and the EPNs communication network, it can also be used to focus on system failures which may occur because of failures in the communication network.

As simple example, we will consider a system consisting of a steady source of energy (e.g. a thermal or nuclear power plant), a source of renewable energy, a storage system (e.g. a dam), and a fast ramping source of energy that is called upon when the energy needs exceed the available energy from the steady source, the renewal energy and the storage system. An imperfect packet communication network is used to inform the different system units, and we use the possible loss of data in the communication network to illustrate the effect of imperfect QoI.

We consider a source of non-renewable (e.g. nuclear or fossil) from which, the system we are considering, is contractually set to receive energy at a rate $c$ units per unit time (e.g. MWatts) which has been selected as a result of energy demand forecasts and of the different system components, such as the storage unit, the renewable energy supply, back-up fast ramp energy devices which are available when all else fails.

\section{A. Modeling the Power System as a G-Network}

Due to other unpredictable needs, our system's energy demand is a random process which makes requests in excess of this value $c$. The excess has a rate of $D$ units per unit time. A RES source (e.g. wind or phovoltaic) generates a random supply of energy units at a rate $\lambda$ which is placed in a storage unit (e.g. a dam, compressed air storage unit, water tower, battery) which can store at most $S$ energy units (e.g. GigaJoules). The stored energy will be used when the demand exceeds the fixed level $c$. The storage unit has a loss rate of $\mu$ through leakage, but in addition, storage of the renewable energy also results in the loss of a fraction of $L$ of the energy during the conversion (e.g. from electricity generated by a renewable source to water that has been pumped into a dam), while extraction of energy from storage also results in a loss of a fraction $l$. The energy from storage cannot be extracted at a rate grater than $\alpha$ since the storage unit (e.g, a dam), has a generator whose maximum energy production rate is $\alpha$. Similarly, if the storage unit is a battery, it has a maximum output current and a fixed voltage, which together will determine the battery's maximum output power. If the storage unit is empty, then we can call upon a source of energy (such as a gas turbine) that generates at rate $\beta$.

\section{The System with Perfect Information}

We first consider the system we have described under perfect conditions regarding tha available information, so that every part of the system behaves correctly and instantaneously based on other events in the system. The mathematical model is based on an EPN, which is a novel queuing analysis of an energy generation, storage and energy consumption system.

The EPN includes a queue $Q R$ which represents the storage of the energy generated by the RES. It has Poisson arrivals of rate $(1-L) \lambda$, the effective arrival rate of EPs to the energy storage centre, and its maximum storage capacity or queue length is $S$. If the storage is full, the incoming flow of renewable energy will "spill over" and be lost.

Another queue $Q D$ with Poisson arrivals of rate $D$ will represent the unsatisfied demand. $Q D$ has Poisson arrivals $\mathrm{f}$ rate $D$ representing the energy demand, and has departures of EPs which represent requests sent to the storage $Q S$ in the form of "negative customers" [10] each of which will in turn deplete an EP from the storage to represent the satisfaction of the request. Note that the conversion loss at the output of the $Q S$ can also be represented via the probability $l$. When $Q S$ is empty, then the fast ramp generator will turn on.

G-network theory leads to the following equations for the stead-state probabilities $Q$ and $r$, that $Q D$ and $Q S$, respectively, contain at least one unit of energy in demand for $Q D$ or stored for $Q S$. Let us first assume that $S=\infty$. Then

$$
Q=\frac{D}{r \alpha(1-l)+(1-r) \beta}
$$

since when the storage is non-empty (probability $r$ ) the demand will be served by the stored energy at rate $\alpha$ multiplied by the probability that a conversion loss does not occur $(1-l)$, while if the storage is empty then the fast ramp generator will be turned on and satisfy the demand at the rate $\beta$. If $S=\infty$ then the probability that the storage is non-empty is given by:

$$
q=\frac{\lambda(1-L)}{Q \alpha+\mu}
$$

because the store is filled by the RES at rate $\lambda(1-L)$ and it is depleted by leakage at rate $\mu$ and by the energy demand at rate $Q \alpha$. Note that the fact that there may be a energy conversion loss at the output of the storage unit does not reduce (quite to the contrary) the rate at which the storage is depleted. If the storage system has finite capacity, then we will have:

$$
r=q \frac{1-q^{S}}{1-q^{S+1}}
$$

When the energy storage capacity is finite $S<+\infty$, we can also readily compute the spillover rate $\Lambda_{s}$ of renewable energy from $r$ :

$$
\Lambda_{s}=\lambda q^{S} \frac{1-q}{1-q^{(S+1)}}
$$

Also, the average amount of stored energy for finite storage capacity is given by:

$$
E_{s}=\frac{q}{1-q}-\frac{(S+1) q^{S}}{1-q^{(S+1)}}
$$

If the storage unit has no leakage we will have $\mu=0$ and the load factor for the RES storage centre is: $\rho=\lambda(1-L) / \alpha$. 
Thus when the energy storage centre has unlimited capacity and no leakage loss we obtain:

$$
\begin{gathered}
Q=\min \left[1, \frac{D}{\beta}+\frac{\lambda(1-L)}{\alpha}-\frac{\lambda(1-L)(1-l)}{\beta}\right] \\
q=\min \left[1, \frac{1}{1+\frac{\alpha}{\beta}\left[\frac{D}{\lambda(1-L)}-(1-l)\right]}\right.
\end{gathered}
$$

with $0 \leq Q \leq 1,0 \leq q \leq 1$.

Thus a system that is set to operate at a satisfactory operating point, should have $Q \approx 0$ so that the excess demand is always satisfied, and enough reserve $q>0$ so that when there is excess demand then there is also enough stored energy so that demand can be rapidly satisfied. With an unbounded storage and no leakage, the average stored energy is:

$$
E=\frac{\beta}{\alpha\left[\frac{D}{\lambda(1-L)}-(1-l)\right]}
$$

provided that $\lambda(1-L)(1-l)<D$, i.e. when the effective rate at which renewable energy is stored is just below the excess demand. Thus for an energy demand rate $d$, the production rate $c$ has to be selected so that it is just set just below the level needed $c=d-\lambda(1-L)(1-l)-\epsilon$ for a very small $\epsilon$ to satisfy demand. The system then operates just below the needed capacity.

\section{THE EFFEct OF POOR QoI}

If the demand exceeds the set generation rate $c$, a message will be sent to the energy storage centre requesting the transfer of energy, and assume that the system has a fine grained control process so that a message needs to be sent for each successive EP that is needed from storage. If the message is delayed this will affect the system. Even more so, if the message is lost or misinterpreted with probability $(1-p)$, this will obviously affect the energy system. If the message does arrive correctly, but the time delay between the instant when the message was sent and the time when the power transfer starts to be made at the storage centre is significant, then we will observe a system disfunction.

\section{A. The Effect of Delayed Information}

If the energy storage centre is empty then the fast rampup generator will also be turned on by sending a message through the communication network with the same probability of message loss and increased total delay for the generator to start producing the required energy. The communication delays will change the system's performance so that the delayed arrival of critical information by themselves modify $\alpha$ and $\beta$ into $\alpha^{\prime}$ and $\beta^{\prime}$, respectively as follows:

$$
\frac{1}{\alpha^{\prime}}=\frac{1}{\alpha}+\frac{1}{\delta}, \quad \frac{1}{\beta^{\prime}}=\frac{1}{\beta}+\frac{1}{\delta}
$$

so that

$$
\alpha^{\prime}=\alpha \frac{\delta}{\alpha+\delta}, \quad \beta^{\prime}=\beta \frac{\delta}{\beta+\delta},
$$

\section{B. Delayed Information with Message Loss}

However, if one deals with a message loss with probability $p$, assuming that an application level end-to-end protocol is used to authenticate arriving messages and then insure that in the energy system that we consider any lost messages are resent by the source and eventually received, the parameter $\delta$ will be modified by the loss of messages as follows:

$$
\frac{1}{\delta^{\prime}}=(1-p) \frac{1}{\delta}+p\left[\frac{1}{\delta^{\prime}}+R\right]
$$

In the above expression, $R$ is some average delay that elapses between the actual loss of a message and the instant that the the source determines that the message has not been correctly received and the message is retransmitted by the source. Furthermore we can imagine that several losses may occur until message is correctly received. Therefore we have:

$$
\frac{1}{\delta^{\prime}}=\frac{1}{\delta}+R \frac{p}{1-p}
$$

so that:

$$
\alpha^{\prime}=\alpha \frac{\delta^{\prime}}{\alpha+\delta}, \quad \beta^{\prime}=\beta \frac{\delta^{\prime}}{\beta+\delta^{\prime}},
$$

This leads to:

$$
Q^{\prime}=\frac{D}{\left[r^{\prime}\left(\alpha^{\prime}(1-l)+\left(1-r^{\prime}\right) \beta^{\prime}\right]\right.}
$$

and the storage system load factor is:

$$
q^{\prime}=\frac{\lambda(1-L)}{Q^{\prime} p \alpha^{\prime}+\mu}
$$

By placing $\alpha^{\prime}$ in the place of $\alpha$ and $\beta^{\prime}$ instead of $\beta$ in all the expressions of Section $\mathrm{V}$, we can estimate the effect of communication message loss and the resulting computer control system delay. For instance, the average value of the stored energy when the energy storage centre has no leakage loss and its capacity is unbounded, is given by:

$$
E^{\prime}=\frac{\beta^{\prime}}{\alpha^{\prime}\left[\frac{D}{\lambda(1-L)}-(1-l)\right]}=E \frac{\delta^{\prime}+\alpha}{\delta^{\prime}+\beta}
$$

so that the net effect of information delay and loss can be studied directly from these expressions.

In particular, when $\delta^{\prime}>>\alpha, \beta$, i.e. when the communication delays are small despite possible message losses, we have:

$$
E^{\prime} \approx E \cdot\left[1+\frac{\alpha-\beta}{\delta^{\prime}}\right]
$$

so that depending on whether $\alpha>\beta$ or not, $E^{\prime}$ will be larger or smaller than $E$ in the presence of communication delays and possible message losses.

\section{CONCLUSIONS}

While conventional sources of electrical power have relied on the physical properties of the electric grid, where characteristics of the network itself such as voltage, current will signal back to the generators when additional power is needed, modern systems rely increasingly on the information obtained from metering at different levels, both to control power flows 
and to determine pricing. In this paper we have focused on the effect that the Quality of Information related to metered data will have on the adequate supply of power. Using an example where an additional source of power together with energy storage are used to compensate for random surges in consumption, we have shown that the degree to which the power network meets the needs of the consumers is directly related to the precision with which the information regarding power consumption can be correctly conveyed to the different system elements. In future work we expect to address the manner in which network attacks [12], [18] can impair the fast adaptive management of energy systems. We also plan to analyse the impact of the performance and quality of service of data networks on the ability of the smart grid to meet the needs of its customers. Future work will also examine how mutiple distinct user classes [5], [9] with different energy needs can together make the best use of smart network management, and whether adaptive techniques initially designed for packet network [17] can also be used to dispatch energy.

\section{REFERENCES}

[1] E. Gelenbe and R. R. Muntz "Probabilistic Models of Computer Systems - Part I (Exact Results)", Acta Informatica, 7: 35-60, 1976.

[2] E. Gelenbe "Diffusion approximations, waiting times and batch arrivals", Acta Informatica, 12: 285-303, 1979.

[3] E. Gelenbe and K. C. Sevcik "Analysis of update synchronization for multiple copy data bases", IEEE Transactions on Computers, vol. 28 no. 10 , pp. 737-747, 1979.

[4] E. Gelenbe and G. Hébrail, A probability model of uncertainty in data bases, International Conference on Data Engineering (ICDE), IEEE Computer Society, pp. 328333, 1986.

[5] V. Atalay and E. Gelenbe, Parallel algorithm for colour texture generation using the random neural network model, International Journal of Pattern Recognition and Artificial Intelligence, vol. 6, no. 2-3, pp. 437446, 1992.

[6] R. Ramanathan, R. Engle, C.W. Granger, F. Vahid-Araghi and C. Brace "Short-run forecasts of electricity loads and peaks", International Journal of Forecasting, 13 (2): 161-174, 1997.

[7] J. Aguilar and E. Gelenbe "Task assignment and transaction clustering heuristics for distributed systems", Information Sciences 97 (1), 199219,1997

[8] J. Toyoda and H. Saitoh "Proposal of an Open-Electric-EnergyNetwork(OEEN) to realize cooperative operations of IOU and IPP", Proceedings of the 1998 International Conference on Energy Management and Power Delivery (EMPD 98), Singapore, 35 March 1998; Volume 1, pp. 218222.

[9] J.-M. Fourneau and E. Gelenbe, Random neural networks with multiple classes of signals, Neural Computation, vol. 11, no. 4, pp. 953963, 1999.

[10] E. Gelenbe and J.-M. Fourneau "G-Networks with resets", Performance Evaluation, 49: 179-192, 2002.

[11] J. W. Taylor "Density forecasting for the efficient balancing of the generation and consumption of electricity", International Journal of Forecasting, 22 (4): 707-724, 2006.

[12] E. Gelenbe and G. Loukas "A self-aware approach to denial of service defence", Computer Networks, 51 (5): 1299-1314, 2007.

[13] M. Black and G. Strbac "Value of bulk energy storage for managing wind power fluctuations", IEEE Transactions on Energy Conversion, 22(1): 197-205, Mar. 2007.

[14] V. Dordonnat, S. Koopman, M. Ooms, A. Dessertaine and J. Collet "An hourly periodic state space model for modelling French national electricity load", International Journal of Forecasting, 24 (4): 566-587, 2008.

[15] I. Sanchez "Adaptive combination of forecasts with application to wind energy", International Journal of Forecasting 24 (4): 679-693, 2008.

[16] E. Gelenbe "Analysis of single and networked auctions", ACM Trans. On Internet Technology, 9(2), May 2009.
[17] E. Gelenbe "Steps toward self-aware networks", Comm. ACM, Vol. 52 (7), pp. 66-75, 2009.

[18] G. Sakellari and E. Gelenbe, "Demonstrating cognitive packet network resilience to worm attacks", Proc. ACM Conference on Computer and Communications Security, pp. 636-638, 2010.

[19] H. Berthold, M. Boehm, L. Dannecker, F.-J. Rumph, T.B. Pedersen, C. Nychtis, H. Frey, Z. Marinsek, B. Filipic and S. Tselepis "Exploiting renewables by request-based balancing of energy demand and supply", Proc. 11th IAEE European Conference, 2010.

[20] Nationalgrid UK 2010. Metered half-hourly electricity demands. Nationalgrid UK. http://www. nationalgrid.com/uk/Electricity/Data/Demand+Data/.

[21] NREL 2010. Wind Integration Datasets. NREL http://www.nrel.gov/wind/ integrationdatasets/.

[22] Randy H. Katz, David E. Culler, Seth Sanders, Sara Alspaugh, Yanpei Chen, Stephen Dawson-Haggerty, Prabal Dutta, Mike He, Xiaofan Jiang, Laura Keys, Andrew Krioukov, Ken Lutz, Jorge Ortiz, Prashanth Mohan, Evan Reutzel, Jay Taneja, Jeff Hsu, Sushant Shankar "An informationcentric energy infrastructure: The Berkeley view", Sustainable Computing: Informatics and Systems 1, pp. 722, 2011.

[23] E. Gelenbe and T. Mahmoodi, "Energy-Aware Routing Protocol in the Cognitive Packet Network", International Conference on Smart Grids, Green Communications, and IT Energy-aware Technologies (Energy 2011), Venice, Italy, 22-27 May 2011, ISBN : $978-1-61208-$ $006-2$.

[24] E. Gelenbe and C. Morfopoulou "A framework for energy aware routing in packet networks", The Computer Journal 54 (6): 850-859, 2011.

[25] E. Bitar, R. Rajagopal, P. Khargonekar, and K. Poolla "The role of co-located storage for wind power producers in conventional electricity markets", Proc. American Control Conference (ACC), 3886-3891, July 2011.

[26] K. Chandy, S. Low, U. Topcu, and H. Xu. A simple optimal power flow model with energy storage. In 49th IEEE Conference on Decision and Control (CDC), 1051-1057, Dec. 2010.

[27] P. Grünewald, T. Cockerill, M. Contestabile, and P. Pearson "The role of large scale storage in a GB low carbon energy future: Issues and policy challenges", Energy Policy, 39(9): 4807-4815, 2011.

[28] H. Oh "Optimal planning to Include Storage Devices in Power Systems", IEEE Transactions on Power Systems. 26 (3): 1118-1128, Aug. 2011.

[29] H.-I. Su and A. E. Gamal "Modeling and analysis of the role of fastresponse energy storage in the smart grid", Proceedings of the FortyNinth Annual Allerton Conference on Communication, Control, and Computing, University of Illinois at Urbana-Champaign, Sept. 2011.

[30] N. Wade, P. Taylor, P. Lang, and P. Jones "Evaluating the benefits of an electrical energy storage system in a future smart grid", Energy Policy, 38 (11): 7180-7188, 2010.

[31] K. Sakai and Y. Okabe, "Quality-aware energy routing toward ondemand home energy networking", Proceedings of 2011 IEEE Consumer Communications an Network Conference; Las Vegas, NV, USA, 912 January 2011; pp. 10411044.

[32] E. Gelenbe. Energy packet networks: Modeling Smart Electricity Storage to Meet Surges in Demand. 5th International Conference on Simulation Tools and Techniques SIMUTOOLS, March 2012, pp. 1-7, ICST (Inst. for Computer Sciences, Social-Informatics and, Telecommunications Engineering) ISBN: 978-1-4503-1510-4.

[33] E. Gelenbe. Energy packet networks: adaptive energy management in the Cloud. CloudCP12 Proc. 2nd Intl Workshop on Cloud Computing Platforms, Article 1, ACM, NY, USA, 2012, ISBN: 978-1-4503-1161-8 doi $10.1145 / 2168697.2168698$

[34] R. Takahashi , T. Takuno and T. Hikihara "Estimation of Power Packet Transfer Properties on Indoor Power Line Channel", Energies 5, 21412149, 2012; doi:10.3390/en5072141 